\section{Wuhan is the booming capital of the eastern inland province of Hubei. The city is investing heavily in research and development and has become China's 'optics valley'.}

\author{
ARTICLE COUNT (AC): 486 \\ FRACTIONAL COUNT (FC): 222 \\ WEIGHTED FRACTIONAL COUNT \\ (WFC): 217
}

$\mathrm{W}$ uhan is an important centre for manufacturing, information technology, transportation and education. The optoelectronics industry in particular has enjoyed rapid growth in recent years; in 2013, Wuhan produced more than 127 million kilometres of fibre optic cables, the largest output of any Chinese city.

In November 2014, Wuhan initiated the eighth instalment of its highly selective '3551 optics valley talent program'. Launched in 2011, the programme aims to recruit global leaders and young researchers to work in five designated areas: information technology, biotechnology, energy and environment, specialized equipment, and a sector that China calls modern service (essentially, IT-enabled service industries). The latest instalment increases the funding available for top researchers to US $\$ 16.3$ million.

Of Wuhan's higher education institutions, Wuhan University (WHU) and Huazhong University of Science and Technology (HUST) are the two largest in the index. Together they account for two-thirds of the city's weighted fractional count (WFC) - a measure of the relative contribution of an institution to the papers it has published. WHU has 154 articles (WFC $=98.8$ ) in the index. The 121-year-old establishment is strongest in chemistry, which accounts for $64 \%$ of its WFC. Aiwen Lei from the department of chemistry was WHU's most prolific chemistry researcher by some distance, having led 20 articles $(\mathrm{WFC}=16.4)$ on the use of free radical coupling reactions in organic synthesis. Lei explains that the reactions can be used to introduce various functional groups onto organic molecules. "The free radicals bind by forming carboncarbon bonds and releasing hydrogen gas."

Hongbing Shu from the college of life sciences was the largest contributor in the life sciences, having published four articles $(\mathrm{WFC}=3.4$ ) on innate immunity. Specifically, Shu studied how enzymes suppress signalling molecules, including tumour necrosis factors and interleukins. The findings have important implications in the development of cancer treatments and for understanding inflammation. Xiangdong Fu from the same college is also a significant contributor to the index, with four articles (WFC $=1.8$ ) - including two in Cell - on cell reprogramming. One of his articles, "Direct conversion of fibroblasts to neurons by reprogramming PTB-regulated RNA circuits", was listed as one of China's 100 most influential academic papers in 2013 by the Institute of Scientific and Technical Information of China.

HUST's research strengths lie in physics and material sciences. Last year, the university had 109 articles $(\mathrm{WFC}=43.6)$ in the index, of which $61(\mathrm{WFC}=25.3)$ were in this field. Xiangshui
Miao and Jingping Xu from the school of optical and electronic information are HUST's largest contributors. Miao published four articles (WFC $=2.9$ ) on ultrathin films, while Xu published two (WFC $=1.5$ ) on metal oxide semiconductors.

Wuhan has four other national key universities in the index: Central China Normal University (CCNU), China University of Geosciences (CUG), Huazhong Agricultural University and Wuhan University of Technology. Each of these institutions contributed between $4 \%$ and $7 \%$ of the city's WFC.

Of CCNU's 43 articles (WFC = 15.9), 84\% were in chemistry. The largest contributor was Anxin Wu from the department of chemistry, with four papers $(\mathrm{WFC}=3.9)$ on drug design and development. "We have made much progress in the synthesis of natural products," says Wu. He adds that 40 natural compounds were made through one-pot synthesis - multiple reactions in a single reactor.

CUG is Wuhan's largest contributor to earth and environmental sciences. This highly specialized institution has $65 \%$ of its WFC in this field. The work was broadly shared, with no CUG researcher contributing to more than one article in the index. CUG does have one wholly authored paper (WFC = 1) in Earth and Planetary Science Letters, led by Yongfeng Wang from the department of geology.

\title{
WUHAN ANALYSIS
}

Wuhan data

Wuhan's large population dilutes its ratios of gross domestic product (GDP) and WFC per person.

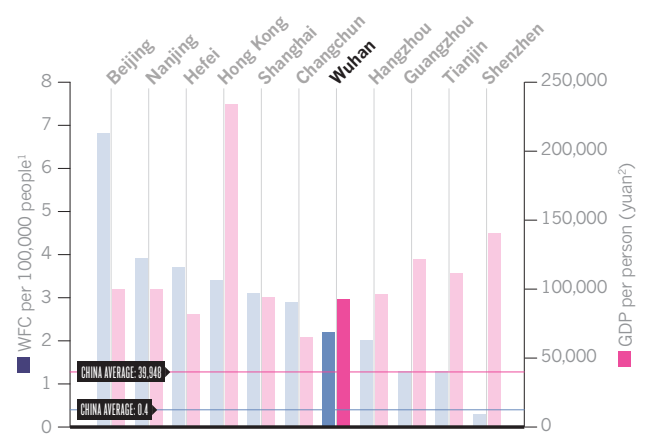

City WFC breakdown

Wuhan has 33 institutions (including CAS) in the index, the third largest after Beijing and Shanghai

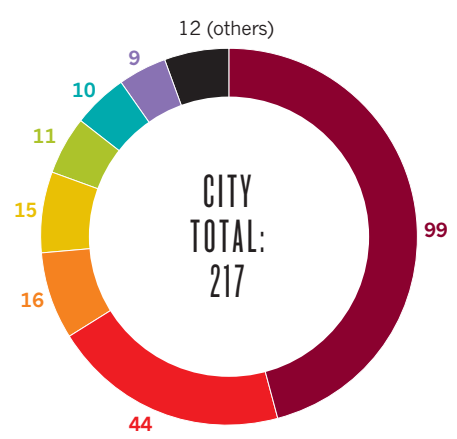

City subject spread

Wuhan's subject spread is similar to

China's overall.

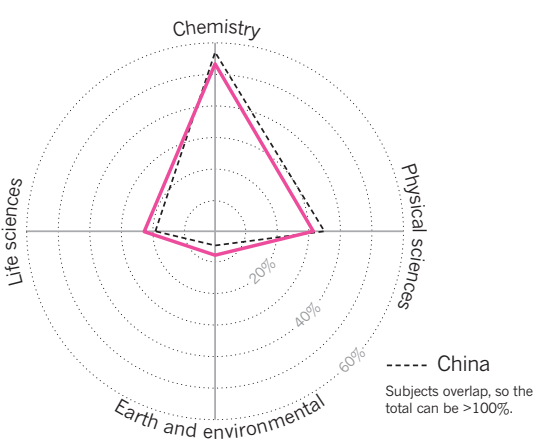

